\title{
Changes in the Contents of the Oral Flora, in Gingival Hypertrophy Caused by Fixed Orthodontic Appliances
}

Ilma Robo ( $\sim$ ilmarobo@yahoo.com )

Albanian University https://orcid.org/0000-0002-8328-375X

Saimir Heta

Pediatric Surgery, QSUT, Tirane, Albania

Enxhi Gjumsi

Private Dental Clinic, Tirane, Albania

Vera Ostreni

Department of Morphology, QSUT, Tirane, ALbania

\section{Research Article}

Keywords: orthodontic fixed apparatus, oral flora, bacterial sensitivity, antibiotic, bacterial culture

Posted Date: July 6th, 2021

DOI: https://doi.org/10.21203/rs.3.rs-474620/v1

License: (a) (i) This work is licensed under a Creative Commons Attribution 4.0 International License.

Read Full License 


\section{Abstract}

Background: The placement of orthodontic apparatus in the oral cavity, according to the literature, should influence the alteration of oral flora, especially the subgingival one. The purpose of the study is to evaluate the subgingival flora of patients with fixed orthodontic appliances, regardless of placement time. In 3 cases of patients with fixed orthodontic appliances, a bacterial sample of the gingival sulcus was taken for laboratory examination. Patients were clinically evaluated for the presence or tendency, of having gingival hypertrophy.

Results: Results from the 3 cases included in the study, 1 of them came up with Streptococcus Anginosus positive, Doxycilin-sensitive. The tendency for gingival hypertrophy was maximal $3 \%$ to $1.5 \%$ respectively in each patient. In the patient with different oral flora, daily topical treatment with tetracycline, placed in the gingival sulcus, was applied.

Conclusions: Alteration of the oral flora with the placement of fixed orthodontic appliances is not a fully verifiable fact, as it indicates the patient's follow-up, at the time of placement of the apparatus and until removal after orthodontic treatment, depending on the 2-3-year period of treatment. The tendency for gingival hypertrophy is apparently high, versus the presence of fixed orthodontic apparatus.

\section{Background}

Gingival hypertrophy, caused by the local irritating effect of parts of the fixed apparatus in the oral cavity, in patients undergoing orthodontic treatment, within the indicated interval of orthodontic treatment, oriented by the established diagnosis, is a common pathology. This is due to the primary fact that the orthodontic appliance makes it difficult to maintain oral hygiene, despite the patient's good will to be careful against this fact. ${ }^{(1,2,3)}$

The study aims to show differences in the oral flora of patients who have a fixed orthodontic appliance in the oral cavity, placed for a considerable period of time; time during which the adaptation of the oral flora also occurred.The fixed orthodontic appliance with all its elements that are placed in the oral cavity, has a smooth surface that is easily cleaned. The situation is complicated as the apparatus increases the surface volume above the tooth surface, versus the vestibule. The first element and the second element of the clinical situation of gingival hypertrophy, lies in the fact that brackets or even bands are glued to the tooth surface with the help of composite and cement. Here is introduced the irritating and initiating element for the creation of the bacterial plaque, as the rough surface on which the bacterial plaque can be created is added. $(2,3,4,5,6,7)$

The primary definition of bacterial plaque involves the fact that it manages to form more rapidly on hard surfaces and begins to deposit initially on the interproximal surfaces.

Another element that makes it difficult for the patient to maintain oral hygiene with the orthodontic appliance in the oral cavity is the covering or interconnection of the teeth with the wire that joins the 
brackets of the apparatus placed in the oral cavity. Thus, the patient can't freely clean the interproximal surfaces of the teeth with a fixed orthodontic appliance. $(3,5,8,9,10,11,12,13,14)$

From the literature data there is no reason to change the oral flora, as both the metallic composition of the apparatus and the inorganic or organic composition of the composite or cement to which the apparatus is fixed are not elements that can be utilized by the constituent bacteria of the oral cavity during the cycle. their vital. Even the cement and adhesive composite for orthodontic appliances is even better and more processed if we compare them with the composite used for dental therapy, or with the cement used for dental prosthetics. $(8,10,15,16,17)$

A single data that has been published in the literature relates to the sensitivity of epithelial cells to nickel, the metal component of orthodontic appliance wires. And that's why gingival hypertrophy caused by orthodontic appliances is not a separate classification or subclassification in the classification of periodontal diseases, both in the old classification and in the new classification. $(1,18,19,20,21,22,23,24)$

Gingival hypertrophy caused by fixed orthodontic appliances has the clinical picture mainly of inflammatory hypertrophy, induced by the local presence of bacterial plaque, hypertrophy with allergic stimulus factor, caused by the nickel composition mainly of fixed apparatus wires and hypertrophy caused by hormone fluctuations from the monthly menstrual cycle, as well as from the period of onset or development of adolescence. Sex hormone fluctuations are known to have a significant effect on gingival margins, mainly as estrogen affects gingival tissue proliferation and progesterone increases angiogenesis. ${ }^{(5,25,26,27,28,29)}$

So, the gingiva becomes the prey of healthy hypertrophic gingival enlargement, as where there is new tissue the blood vessels are also added. The combination of systemic conditioners is healthy, only when bacterial plaques are absent. As in the presence of bacterial plaque the clinical situation changes towards pathology and the "healthy" gingival hypertrophy turns into pathology, which in some cases is combined with the allergy to the metal constituent products of the orthodontic appliance. $(8,30,31,32,33,34)$

Oral flora has its usual ingredients, in content and composition ratios. The minimal change, either in the new constituent element or in the increase of the number of the existing element of the normal oral flora in the gingiva, shows significant changes of inflammation. However, the growth and microbiological cultivation of oral flora is specific and relatively costly in general, and in Albania in particular. In our country there is also the difficulty in cultivating special voices of the oral flora, mainly anaerobes specific constituents of the oral flora, causes of periodontal pathologies with specific clinical appearance.

$(12,36,37,38)$

\section{Methods}

The study was applied at the Dental Clinic of Albanian University, in the period December 2019 - February 2020. The study is of the case report type of 3 cases of selected ad-hoc patients, with orthodontic 
appliance fixed in the oral cavity.

Patients were included in the study after verbal confirmation and signing of the consent consent for the readiness of data collection indicated to meet the purpose of the study. Patients were recorded demographic data and data about existing systemic diseases or not, in patients. The existence of systemic diseases affects the onset of gingival hypertrophy, which is caused by three classes of medications that patients can take to treat systemic diseases such as: hypertension, epilepsy, and situations where immunosuppression is indicated. Taking medication for these clinical situations affects the occurrence of gingival hypertrophy regardless of whether or not they have orthodontic apparatus in the oral cavity.

The patients were all three females, with higher education, in the age range $20-25$ years. Patients were selected ad-hoc and this similarity in the three data collected simplifies the fulfillment of the purpose of the study, as under the same conditions we expect to control only the effect of the presence of bacterial plaque, and consequently the level of personal hygiene, on the appearance of gingival hypertrophy.

Patients were evaluated for the presence of gingival hypertrophy and underwent analysis of the oral flora of the gingival sulcus at the teeth with fixed orthodontic apparatus. ${ }^{(3,6,8,12,22,39)}$

Consent in the participation of patients in the study was performed with the signature of the patients themselves.

\section{Results}

\section{Patient no.1: E. Gj.}

23-year-old patient, E. Gj. was treated with the aim of directing the teeth, applying the orthodontic technique with fixed orthodontic apparatus in both arcades. This treatment started 1 year ago and throughout this time period has followed the stages of orthodontic activation.

During the examination it was noticed that she has 25 teeth present in the oral cavity; this is because the third molar teeth in both jaws respectively: $18,28,38,48$ have not erupted, the maxillary canine (23) is impacted and the first maxillary premolars $(14,24)$ have been extracted as indicated by orthodontic treatment that the patient has been undergoing for 1 year.

Periodontal status: Healthy fixed gingiva with high fibrocytic tendency. The width of the fixed gingiva is classified as high respecting the narrowing zones of width, respectively in the frenulum areas. Strong fibrotic median frenules. The maxillary median frenulum fixed very low, has even disappeared the interdental papilla between the two incisors. The interdental papilla appears partially absent under the class 3 classification for papillary height. Marginal gingiva with tendon predominantly papillary in the area of the mandibular incisors. These data are presented in Fig. 1. The index of bacterial plaque is 
relatively low, mainly in the mandibular incisors on the vestibular surfaces, where the tendency for marginal gingivitis in the papillary areas is even higher. Data presented in Fig. 2.

Gingival hypertrophy index: Tendency to gingival hypertrophy, vertical and horizontal index of gingival hypertrophy were in values $\leq 3 \mathrm{~mm}$. The way of data registration is shown in figure no.3. Inflammation of soft tissues may come as a result of persistent force exerted by orthodontic braces; followed by this clinical situation by the tendency for inflammatory hypertrophy.

Using an absorbent to obtain the necessary material, which was then sent to the laboratory. Figure 5 shows the gingival fluid sampling technique and the laboratory analysis sheet performed.

From the test results, the patient tested positive for Streptococcus Anginosus, with sensitivity to various antibiotics such as: Amikacin, Cefazolin, Cefixime, Cefotaxime, Cefoxitin, Ceftazidime, Ceftriaxone, Ciprofloxacin, Clarithromycin, Doxycycin, Eryxamine, Pexycycin, Ery Trimethoprim / sulfamethoxazole.

\section{Patient no.2: A. Ç. -25 years old}

The patient was presented to the University Dental Clinic in order to obtain the appropriate data for the study. After performing the anamnesis, it was found that the patient had 1 year and 4 months that she was equipped with orthodontic apparatus in both arcades and that she was suffering from heart failure.

In the clinical examination the presence of 28 teeth was noticed, as 18,28,38,48 were extracted with the open technique, ie with surgical procedure in order for the orthodontic treatment to be more successful, while in the laboratory examination oral flora was observed. normal.

Periodontal status: Presence of periodontal stone in the interdental spaces maxillary and mandibular incisors. Data shown in Fig. 6.

The patient presents pink gingiva, not erythematous.

Local gingival hypertrophy presented locally in areas 42.43 and 23.24, documented in Fig. 7.

The patient has good hygiene. On the lingual side there is no presence of bacterial plaque. Obtaining the material for a further laboratory examination using buffer and absorbent, accompanied by the analysis sheet are shown in Fig. 9.

Patient no.3: Q. L. - 21 years old

Patient no.3 Q. L. is under orthodontic treatment for 1 year. She has the orthodontic appliance present only in the maxillary arcade. Examination showed the absence of 2 premolars of $1(14,24)$ and the presence of another 30 teeth. The patient after laboratory examination, resulted in normal oral flora. The patient has a pink gingiva. Presence of a maxillary medial frenulum with appendix. Tendency to local gingival hypertrophy in the area of 12.13 and yes tendency to local gingival hypertrophy: 15 (mesial / distal). The above data are shown in Fig. 10. 
Tendency to local gingival hypertrophy: $22.25(\mathrm{~m} / \mathrm{d})$. Oral hygiene more favorable compared to the maxillary arcade because braces make it difficult. The placement of the adsorbent for obtaining the material is shown in Fig. 10, accompanied by the analysis sheet.

\section{Discussions}

Recently, orthodontic treatment is being widely adapted for both adolescents and adults. The application of orthodontic appliances in addition to helping to correct the position of the teeth and achieve the desired aesthetics, can also improve gingival health. This is based on the hypothesis raised by Poulton and Lang who after their study, showed positive effects on all perioral structures after orthodontic treatment, but on the other hand the second hypothesis is based on the fact that orthodontic treatment makes it difficult to maintain hygiene deteriorating periodontal condition. $(40,41,42,43,44,45,46)$

The use of braces on the labial or lingual / palatal surfaces of the teeth allows the accumulation of bacterial plaque, therefore oral hygiene is a key factor for successful treatment. In the study conducted at the University Dental Clinic, all three cases presented with local gingival hypertrophy. This result is consistent with that of S. Zachrisson\& B.U Zachrisson in 1972, who reported that even if patients have very good hygiene, they can still exhibit gingival hypertrophy, also consistent with data by Surlin et. al, where patients may have favorable hygiene and hypertrophy but no signs of gingival inflammation. $(44,45,46)$

Based on these results, it is concluded that gingival hypertrophy can occur in any patient, whether careful or not with hygiene. But on the other hand in a study conducted at King Khalid University, patients who had good oral hygiene and used floss more than 3 times a day showed no signs of gingival hypertrophy, while those who used brush and floss only once a day, presented the highest\% of grade 2 hypertrophy. The age group of patients with a higher frequency of hypertrophy in this study was 10-19 years old. $(45,46,47)$

This data is compatible with that of Boydet.et.al where according to this source, oral hygiene is better in adults than in adolescents because the decision of adults for treatment is not influenced by other persons such as parents or their peers.

Orthodontic treatment may or may not affect the oral flora. Based on our study, only 1 case tested positive for Streptococcus anginosus while other patients resulted in normal oral flora, concluding that orthodontic treatment does not necessarily have to change the normal flora because it depends more on the immunity of the affected individual. There is mixed evidence that there is no need for changes in the patient's individual oral flora, just as there is evidence that authors such as Sakamaki and Bahn rely on the idea that lactobacilli are actively grown in fixed apparatus accompanied by S.mutans or as

Schglenhauf who reports that fixed devices carry more S.mutans than mobile ones. ${ }^{(46,47,48)}$

Analyzes of the oral bacterial flora in patients who have a fixed orthodontic appliance have been performed while evaluating the periodontal indices with their respective changes. One of these studies 
even takes the analysis further by applying it to large number of patients, which is positive for finding possible correlations. Based on this study, this correlation depends on the time of placement of the fixed orthodontic appliance in the oral cavity. ${ }^{(49)}$

Another interesting direction of the analysis of the oral flora in patients with orthodontic apparatus is brought by the next study ${ }^{(49)}$ where the aim is to find possible changes of the oral flora in the sulcus of patients who have fixed orthodontic apparatus or mobile orthodontic apparatus. The 2012-2013 study is performed on a relatively high number of patients. None of the patients taken in the study was positive for periodontal anaerobes. Patients with mobile devices exhibit better oral hygiene, smaller plaque counts, and fewer inflammatory gingival reactions. ${ }^{(50)}$

Another point of view that has attracted attention for publications in the literature is the tendency of patients towards the appearance of carious lesions, towards the placement of the lingual or vestibular orthodontic apparatus. The study where Wilcoxon and Mann Whitney $U$ tests were used to evaluate qualitative data differences and Fisher's Chi-square tests to evaluate quantitative data between groups while Mc Nemar was used for differences between groups. Based on this study, patients with lingual orthodontic appliances had greater plaque presence 4 and 8 weeks after placement, high rate of gingival inflammation, and more S. mutans present, 8 weeks after apparatus adaptation. No differences were found between the 2 groups regarding salivary flow rate, salivation saturation capacity and Lactobacillus. (51)

Perhaps it is the material or type of bracket structure that affects the pathogenic periodontal microbial colonization and halitosis. This was the aim of another study (52) conducted at Suleyman Demirel University, where 46 patients were selected for fixed orthodontic treatment, age range $11-16$ years. ${ }^{(52)}$

Orthodontic appliances can predispose to the spread of Candida in the oral cavity. The results do not lead to the conclusion that orthodontic appliances can change non-Candida carriers into carriers. Of particular importance is oral hygiene in both patients with fixed and mobile devices. ${ }^{53,54)}$

Regarding the oral flora planted from the samples taken in patients with fixed orthodontic appliances in the oral cavity, studies support the presence of Enterococcus faecalis, E. faecium, Staphylococcus aures, Escherichia Coli and different strains from the Candida group, compared to those use mobile or nonmobile devices. ${ }^{(55)}$ This study concluded that orthodontic appliances alter oral health and have effects on colonizing oral biofilm with the possibility of their spread to various tissues and organs.

Fixed orthodontic appliances have brackets, which make the patient more prone to inflammation, due to the accumulation of biofilm and the inability to have maximum oral hygiene. Another irritating factor in these patients is the use of nickel. In the study patients ${ }^{(56)}$ it was observed that only a small number used the dental floss every day when the treatment was long-term and this is related to the fact that patients undergoing long-term treatment reduce their motivation for oral care and the presence of orthodontic appliance makes floss use difficult. 


\section{Conclusions}

Gingival hypertrophy is caused by various factors, including orthodontic appliances. Although there is no specific classification, this type of hypertrophy may be part of the subclassification of chronic gingival hypertrophy.

The placement of fixed orthodontic appliances compromises oral hygiene, due to the presence of braces, which make it difficult to maintain optimal hygiene and allow the accumulation of bacterial plaque.

Gingival hypertrophy from orthodontic appliances can be localized or generalized. Not all patients undergoing orthodontic treatment have signs of hypertrophy or alteration of the oral flora, although a good proportion of them undergo this phenomenon.

\section{Abbreviations}

Not applicable

\section{Declarations}

\section{Ethics declarations}

\section{Ethics approval and consent to participate}

As the authors of the article, we state that there is no violation of the code of ethics during the realization of this article. Consent in the participation of patients in the study was performed with the signature of the patients themselves, procedures based on national protocols.

\section{Consent for publication}

Accepted.

\section{Availability of data and materials}

The datasets analyzed during the current study are available from the corresponding author.

\section{Competing interests}

The authors declare that they have no competing interests.

\section{Funding}

Not applicable. No funding for this research.

\section{Contributions}


IR collected the scientific data and wrote the manuscript. SH revised and edited the manuscript. Literature research was conducted by SH. EGJ and VO collected the scientific data. All authors read and approved the final manuscript.

\section{Corresponding author}

Correspondence to Ilma Robo, email address: ilmarobo@yahoo.com

\section{Acknowledgements}

Acknowledgments belong to our family. Henri and Hera are Our motivation for further in the field of scientific research.

\section{References}

1. Carranza - Clinical periodontology; Mosby 2009; KapitulliGingivitiDeskuamativ.

2. ScottFroum, DDS; Top causes of gingival enlargement and treatment options"; PerioImplantAdvisory; July 10, 2017.

3. Barbara Anne Taylor; "Management of drug-induced gingival enlargement”; Aust Prescr2003;26:1131 Jan 2003DOI: 10.18773/austprescr.2003.007.

4. Lina M Mejia, DDS, MPH; “Drug-Induced Gingival Hyperplasia”; Updated: Dec 09, 2016.

5. Jingzhi Ma, Ming Li, Yingguang; "Tretatment of gingival hyperplasia induced by cyclosporine A"; May 3, 2011.

6. Seymour RA, Smith DG, Turnbull DN.; "The effects of phenytoin and sodium valproate on the periodontal health of adult epileptic patients."; J Clin Periodontol. 1985 Jul;12(6):413-9.

7. Gelfand EW, Cheung RK, Grinstein S, Mills G. Characterization of the role for calcium influx in nitrogen-induced triggering of human T-cells. Identification of calcium-dependent and calciumindependent signals. Eur J Immunol. 1986;16:907-11. [PubMed].

8. Lederman D, Lumerman H, Reuben S, Freedman PD. Gingival hyperplasia associated with nifedipine therapy. Oral Surg Oral Med Oral Pathol. 1984;57:620-2. [PubMed].

9. Margiotta V, Pizzo I, Pizzo G, Barbaro A. Cyclosporine and nifedipine induced gingival overgrowth in renal transplant patients: Correlations with periodontal and pharmacological parameters, and HLAantigens. J Oral Pathol Med. 1996;25:128-34. [PubMed].

10. Petra Surlin, Anne Marie Rauten, Mihai Raul Popescu, Constantin Daguci, Maria Bogdan; " Periodontal Changes and Oral Health"; Emerging Trends in Oral Health Sciences and Dentistry; March 11th 2015; DOI: $10.5772 / 59248$.

11. Josefsson E, Tarkowski A, Carlsten H. Anti-inflammatory properties of estrogen. I. In vivo suppression of leukocyte production in bone marrow and redistribution of peripheral blood neutrophils. Cell Immunol;142:67-78, 1992. 
12. Pack ARC, Thomson ME. Effects of topical and systemic folic acid supplementation on gingivitis in pregnancy. J Clin Periodontol;7:402-414, 1980.

13. Jammula Surya Prasanna,ChintaSumadhura,ParupalliKarunakar; "A Comparative Analysis of Preand Postmenopausal Females with Periodontitis and Its Response to a Non Invasive Clinical Approach"; J Menopausal Med. 2017 Dec;23(3):202-209.

14. Afri, Zeba\& Bhardwaj, Ashu\& Sawai, Madhuri \& Sultan, Nishat. (2015). Influence of female sex hormones on periodontium: A case series. Journal of Natural science, Biology and Medicine. 6. S1469. 10.4103/0976-9668.166124.

15. Jaishree Tukaram Kshirsagar, Balamurugan;" Role of sex hormones in periodontium during pregnancy: Areview"; International Journal of Applied Dental Sciences 2018; 4(4): 168-173ISSN Print: 2394-7489ISSN Online: 2394-7497IJADS 2018; 4(4): 168-173.

16. Smadi L, Zakaryia A. The association between the use of new oral contraceptive pills and periodontal health: A matched case-control study. J Int Oral Health 2018;10:127-31.

17. Sadeq A, Risk JM, Pender N, Higham SM, Valappil SP. Evaluation of the co-existence of the red fluorescent plaque bacteria P. gingivalis with S. gordonii and S. mutans in white spot lesion formation during orthodontic treatment. PhotodiagnosisPhotodynTher. 2015 Jun;12(2):232-7. doi: 10.1016/j.pdpdt.2015.03.001. Epub 2015 Mar 23. PubMed PMID: 25813147.

18. Lombardo L, Ortan YÖ, Gorgun Ö, Panza C, Scuzzo G, Siciliani G. Changes in the oral environment after placement of lingual and labial orthodontic appliances. Prog Orthod. 2013 Sep 11;14:28. doi: 10.1186/2196-1042-14-28. PubMed PMID: 24326120; PubMed Central PMCID: PMC4384913.

19. Nalçacı R, Özat Y, Çokakoğlu S, Türkkahraman H, Önal S, Kaya S. Effect of bracket type on halitosis, periodontal status, and microbial colonization. Angle Orthod. 2014 May;84(3):479-85. doi: 10.2319/061913-461.1. Epub 2013 Nov 4. PubMed PMID: 24188122.

20. Grudianov Al, Dmitrieva NA, Bulygina VV, Kurchaninova MG. [Changes of gingival groove microbiosis by orthodontic treatment]. Stomatologiia (Mosk). 2012;91(3):61-4. Russian. PubMed PMID: 22968617.

21. van Gastel J, Teughels W, Quirynen M, Struyf S, Van Damme J, Coucke W, Carels C. Longitudinal changes in gingival crevicular fluid after placement of fixed orthodontic appliances. Am J Orthod Dentofacial Orthop. 2011 Jun;139(6):735-44. doi: 10.1016/j.ajodo.2009.10.043. PubMed PMID: 21640879.

22. Alves PV, Alviano WS, Bolognese AM, Nojima LI. Treatment protocol to control Streptococcus mutans level in an orthodontic patient with high caries risk. Am J Orthod Dentofacial Orthop. 2008 Jan;133(1):91-4. doi: 10.1016/j.ajodo.2006.03.031. PubMed PMID: 18174078.

23. Mattingly JA, Sauer GJ, Yancey JM, Arnold RR. Enhancement of Streptococcus mutans colonization by direct bonded orthodontic appliances. J Dent Res. 1983 Dec;62(12):1209-11. PubMed PMID: 6361082.

24. Sun F, Ahmed A, Wang L, Dong M, Niu W. Comparison of oral microbiota in orthodontic patients and healthy individuals. MicrobPathog. 2018 Oct;123:473-477. doi: 10.1016/j.micpath.2018.08.011. 
Epub 2018 Aug 8. PubMed PMID: 30096429.

25. Robo I., I. Marko, J. Taga, V. Ostreni, "Gingiva fikse, impaktimbiperiodont"; Konferenca e 8-të Stomatologjike, organizuarnga Albanian University, 31.03.2012, Libri iabstraktevefq. 16.

26. Y. Kim, E. Kwon, Y. Cho, J. Lee, S. Kim, and J. Choi, “Changes in the vertical position of interdental papillae and interseptal bone following the approximation of anterior teeth," The International Journal of Periodontics \& Restorative Dentistry, vol. 34, no. 2, pp. 219-224, 2014.

27. D. P. Tarnow, A. W. Magner, and P. Fletcher, "The effect of the distance from the contact point to the crest of bone on the presence or absence of the interproximal dental papilla," Journal of Periodontology, vol. 63, no. 12, pp. 995-996, 1992.

28. Y.-J. Wu, Y.-K. Tu, S.-M. Huang, and C.-P. Chan, "The influence of the distance from the contact point to the crest of bone on the presence of the interproximal dental papilla," Chang Gung Medical Journal, vol. 26, no. 11, pp. 822-828, 2003.

29. C. Ghezzi, S. Masiero, M. Silvesth, G. Zanotti, and G. Rasperini, "Orthodontic treatment of periodontally involved teeth after tissue regeneration," International Journal of Periodontics \& Restorative Dentistry, vol. 28, no. 6, pp. 559-567, 2008.

30. F. B. Zanatta, T. M. Ardenghi, R. P. Antoniazzi, T. M. P. Pinto, and C. K. Rösing, “Association between gingival bleeding and gingival enlargement and oral health-related quality of life (OHRQoL) of subjects under fixed orthodontic treatment: a cross-sectional study," BMC Oral Health, vol. 12, no. 1, article 53, 2012.

31. H. A. Eid, H. A. M. Assiri, R. Kandyala, R. A. Togoo, and V. S. Turakhia, "Gingival enlargement in different age groups during fixed orthodontic treatment," Journal of International Oral Health, vol. 6, no. 1, pp. 1-4, 2014.

32. J. S. Kloehn and J. S. Pfeifer, "The effect of orthodontic treatment on the periodontium," Angle Orthodontist, vol. 44, no. 2, pp. 127-134, 1974.

33. S. Zachrisson and B. U. Zachrisson, "Gingival condition associated with orthodontic treatment," The Angle Orthodontist, vol. 42, no. 1, pp. 26-34, 1972.

34. P. Şurlin, A.-M. Rauten, D. Pirici, B. Oprea, L. Mogoantă, and A. Camen, "Collagen IV and MMP-9 expression in hypertrophic gingiva during orthodontic treatment," Romanian Journal of Morphology and Embryology, vol. 53, no. 1, pp. 161-165, 2012.

35. U. K. Gursoy, O. Sokucu, V.-J. Uitto et al., "The role of nickel accumulation and epithelial cell proliferation in orthodontic treatment-induced gingival overgrowth," European Journal of Orthodontics, vol. 29, no. 6, pp. 555-558, 2007.

36. C. Marchese, V. Visco, L. Aimati et al., "Nickel-induced keratinocyte proliferation and up-modulation of the keratinocyte growth factor receptor expression," Experimental Dermatology, vol. 12, no. 4, pp. 497-505, 2003.

37. C. A. Pazzini, L. S. Marques, M. L. Marques, G. O. J. Nior, L. J. Pereira, and S. M. Paiva, “Longitudinal assessment of periodontal status in patients with nickel allergy treated with conventional and nickelfree braces," The Angle Orthodontist, vol. 82, no. 4, pp. 653-657, 2012. 
38. C. Maspero, L. Giannini, G. Galbiati, F. Nolet, L. Esposito, and G. Farronato, "Titanium orthodontic appliances for allergic patients," Minerva Stomatologica, vol. 63, no. 11-12, pp. 403-410, 2014.

39. M. C. G. Pantuzo, E. G. Zenóbio, H. D. A. Marigo, and M. A. F. Zenóbio, "Hypersensitivity to conventional and to nickel-free orthodontic brackets," Brazilian Oral Research, vol. 21, no. 4, pp. 298302, 2007.

40. J. Kurol, A. Ronnerman, and G. Heyden, "Long-term gingival conditions after orthodontic closure of extraction sites. Histological and histochemical studies," European Journal of Orthodontics, vol. 4, no. 2, pp. 87-92, 1982.

41. P. B. Robertson, L. D. Schultz, and B. M. Levy, "Occurrence and distribution of interdental gingival clefts following orthodontic movement into bicuspid extraction sites," Journal of Periodontology, vol. 48, no. 4, pp. 232-235, 1977.

42. C. Reichert, L. Gölz, C. Dirk, and A. Jäger, "Retrospective investigation of gingival invaginations: Part I: clinical findings and presentation of a coding system," Journal of Orofacial Orthopedics, vol. 73, no. 4, pp. 307-316, 2012.

43. A. L. R. Circuns and J. F. C. Tulloch, "Gingival invagination in extraction sites of orthodontic patients: their incidence, effects on periodontal health, and orthodontic treatment," American Journal of Orthodontics, vol. 83, no. 6, pp. 469-476, 1983.

44. L. Gölz, C. Reichert, C. Dirk, and A. Jäger, "Retrospective investigation of gingival invaginations: part II: microbiological findings and genetic risk profile," Journal of Orofacial Orthopedics, vol. 73, no. 5, pp. 387-396, 2012.

45. P. Diedrich and H. Wehrbein, "Orthodontic retraction into recent and healed extraction sites. A histologic study," Journal of Orofacial Orthopedics, vol. 58, no. 2, pp. 90-99, 1997.

46. Angelina G., Giorgio P., Anna B., Giampietro, F., \& Giulio R (2016); "Impact of orthodontic treatment on periodontal tissues: a narrative review of multidisciplinary literature"; Internagtional Journal of Dentistry, 9.

47. Hossam, A., hassan, A., Reena, K., Rafi, A., \& Viral,S (2014); "Gingival enlargment in different age groups during fixed orthodontics treatment"; Journal of International Oral health, 1-4.

48. Riddhi C., Shetty, K., Prakash, A., Rathore, A., \& Sarocj, S (2018); “Orthodontics and oral microflora: synergism or parasitism"; JBR Journal of Interdisciplinary Medicine and Dental Science, 6:234.

49. Guo L, Feng Y, Guo HG, Liu BW, Zhang Y. Consequences of orthodontic treatment in malocclusion patients: clinical and microbial effects in adults and children. BMC Oral Health. 2016 Oct 28;16(1):112. PubMed PMID: 27793138; PubMed Central PMCID: PMC5084385.

50. Abbate GM, Caria MP, Montanari P, Mannu C, Orrù G, Caprioglio A, Levrini L. Periodontal health in teenagers treated with removable aligners and fixed orthodontic appliances. J OrofacOrthop. 2015 May;76(3):240-50. doi: 10.1007/s00056-015-0285-5. PubMed PMID: 25929710.

51. Lombardo L, Ortan YÖ, Gorgun Ö, Panza C, Scuzzo G, Siciliani G. Changes in the oral environment after placement of lingual and labial orthodontic appliances. Prog Orthod. 2013 Sep 11;14:28. doi: 10.1186/2196-1042-14-28. PubMed PMID: 24326120; PubMed Central PMCID: PMC4384913. 
52. Nalçacı R, Özat Y, Çokakoğlu S, Türkkahraman H, Önal S, Kaya S. Effect ofbracket type on halitosis, periodontal status, and microbial colonization. Angle Orthod. 2014 May;84(3):479-85. doi: 10.2319/061913-461.1. Epub 2013 Nov 4. PubMed PMID: 24188122

\section{Figures}

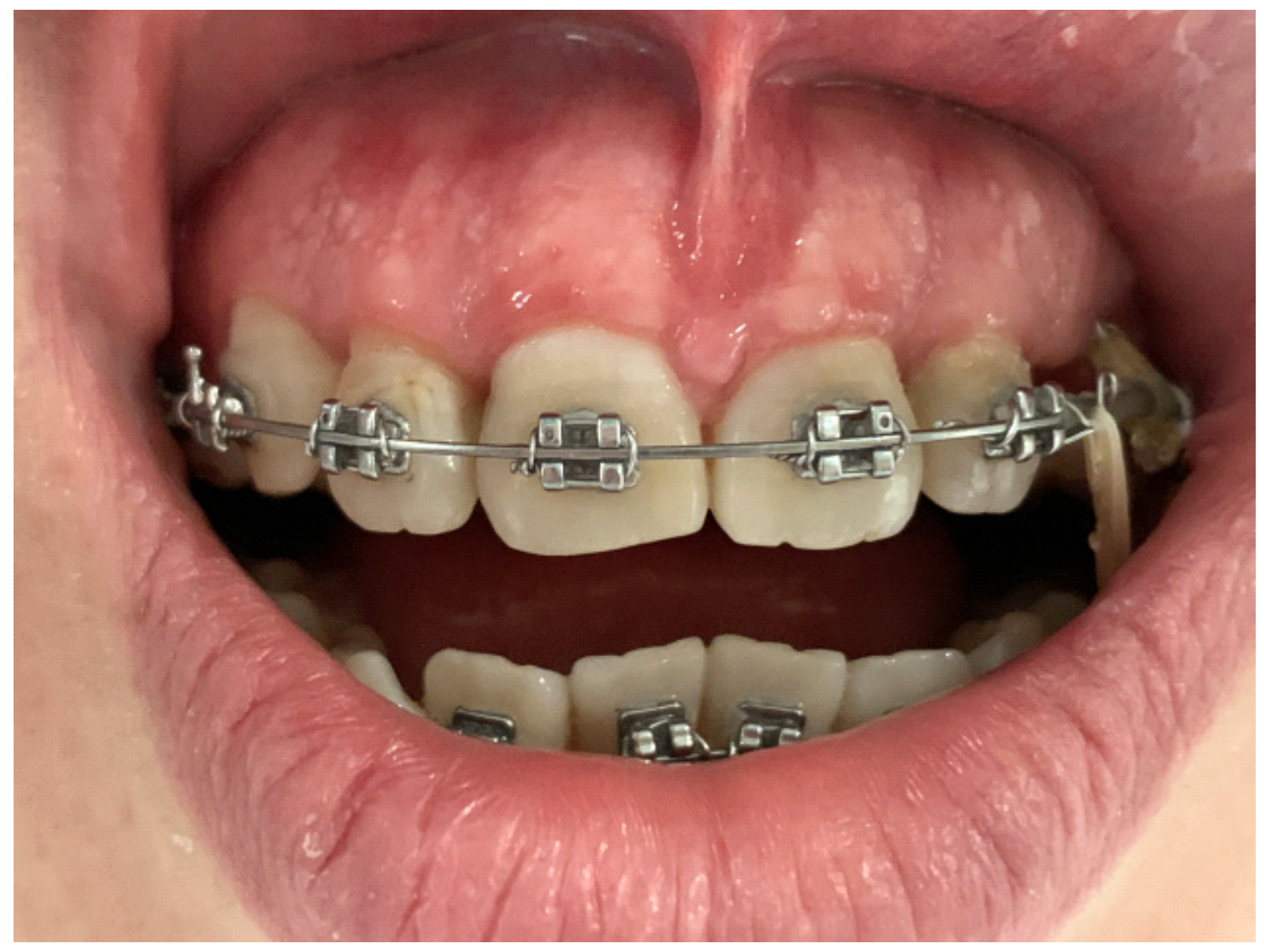

\section{Figure 1}

Patient no.1, photo of the anterior maxilla, showing the maxillary incisors, fixed orthodontic apparatus and fixed gingiva with a width higher than the norm and mainly in pink. 


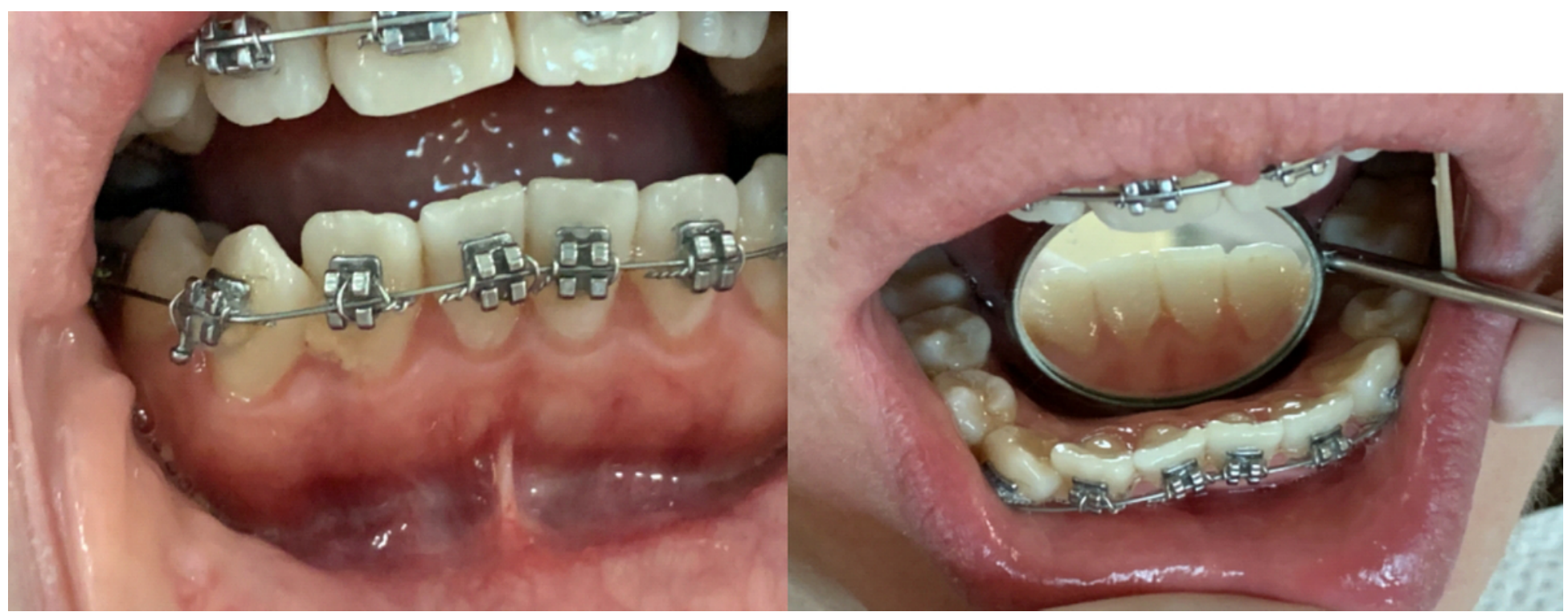

\section{Figure 2}

View of the mandibular, vestibular and lingual incisors, patient no.1, where the presence of bacterial plaque is distinguished, mainly in the interdental areas. On the linguistic side, the patient does not result in the presence of bacterial plaque. 


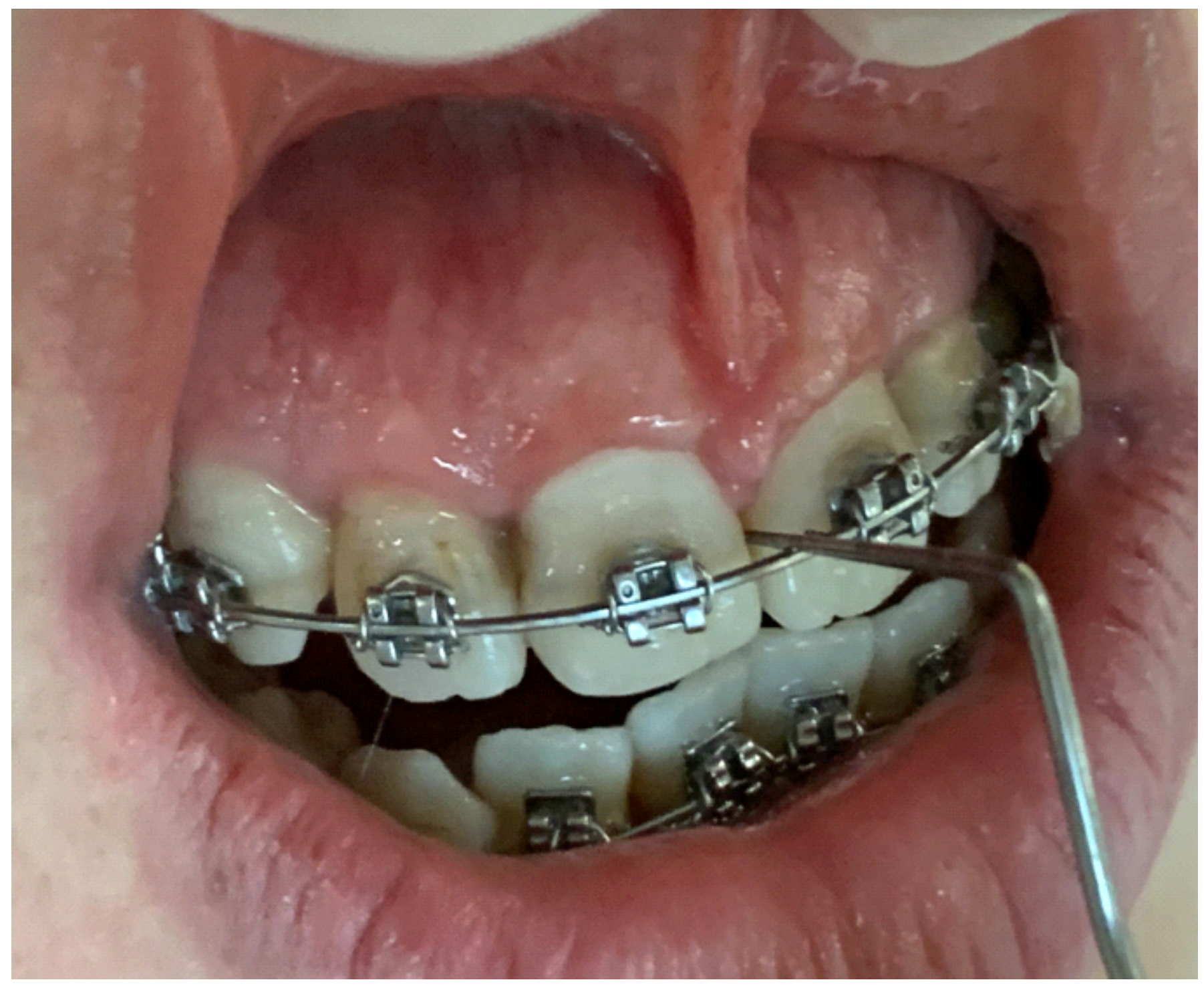

Figure 3

Photo showing exactly how the gingival hypertrophy was assessed, where the vertical and horizontal indices were less than $3 \mathrm{~mm}$. 


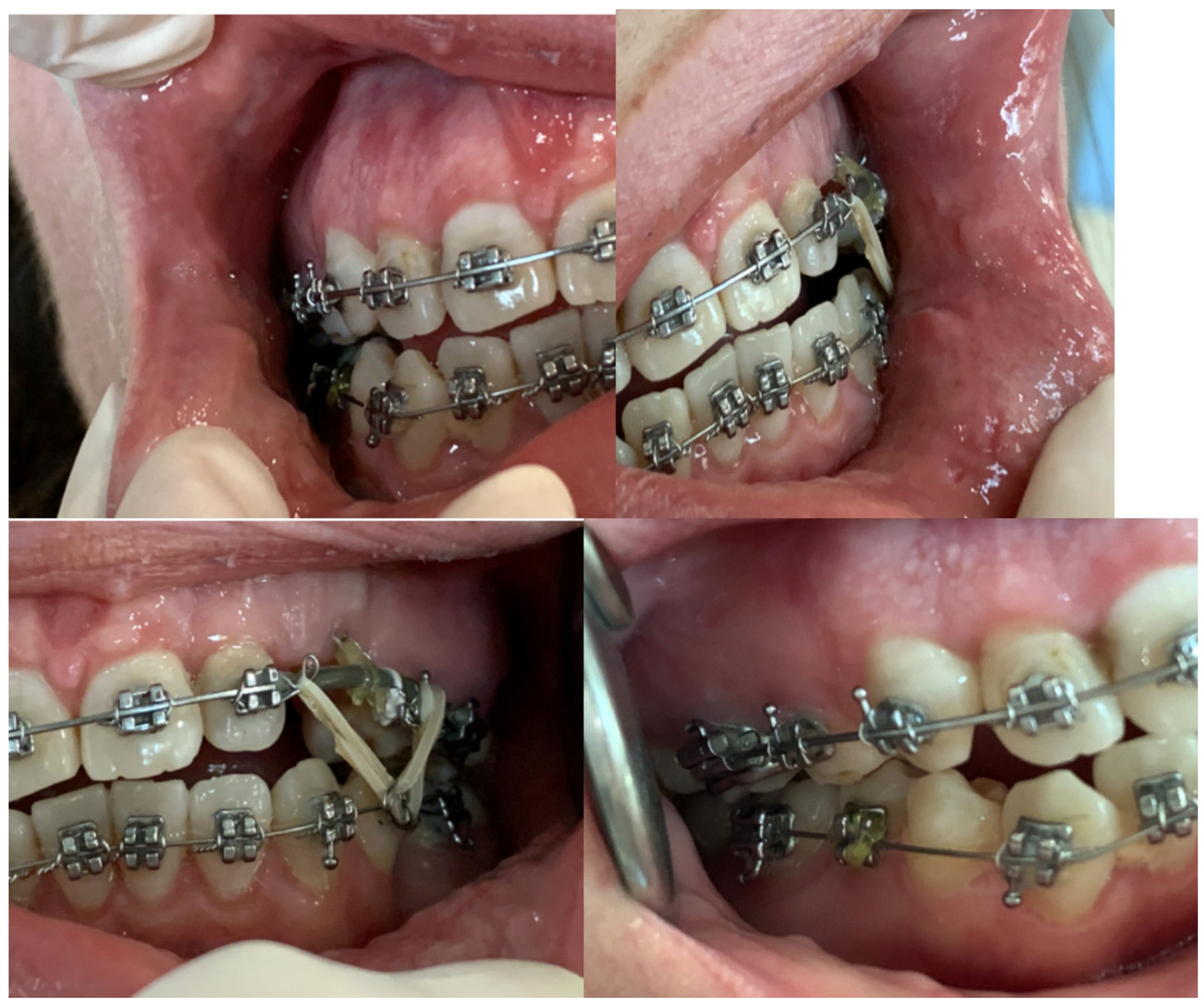

Figure 4

Photo showing the presence of marginal gingivitis through the noticeable change of colors from the antomic and physiological pink color of the gingiva, to the cherry red color. 

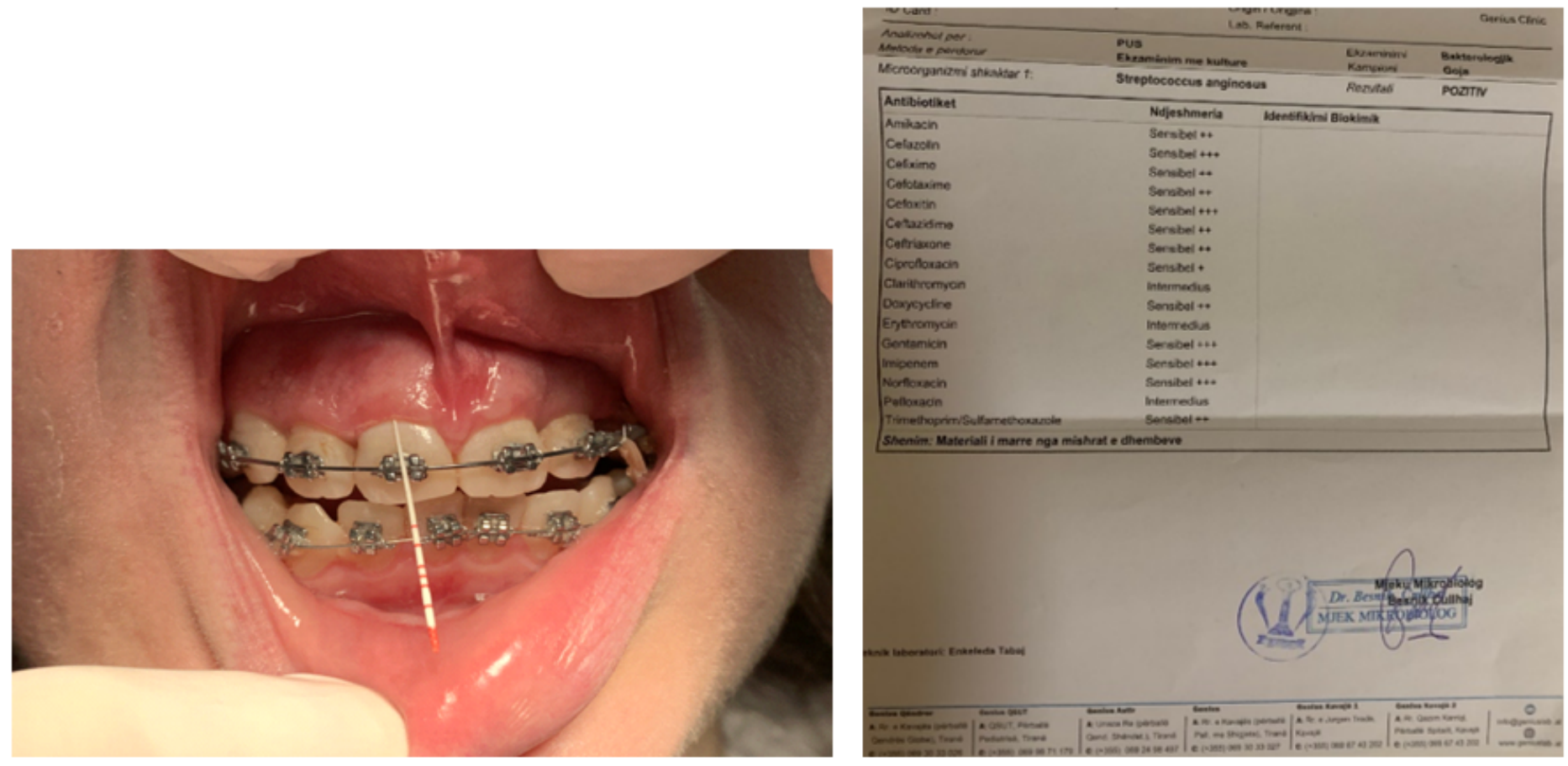

\section{Figure 5}

Absorbent paper placed in the gingival sulcus to take the gingival fluid sample and the sample analysis sheet showing the results in specific names of bacteria, accompanied by sensitivity to antibiotics.

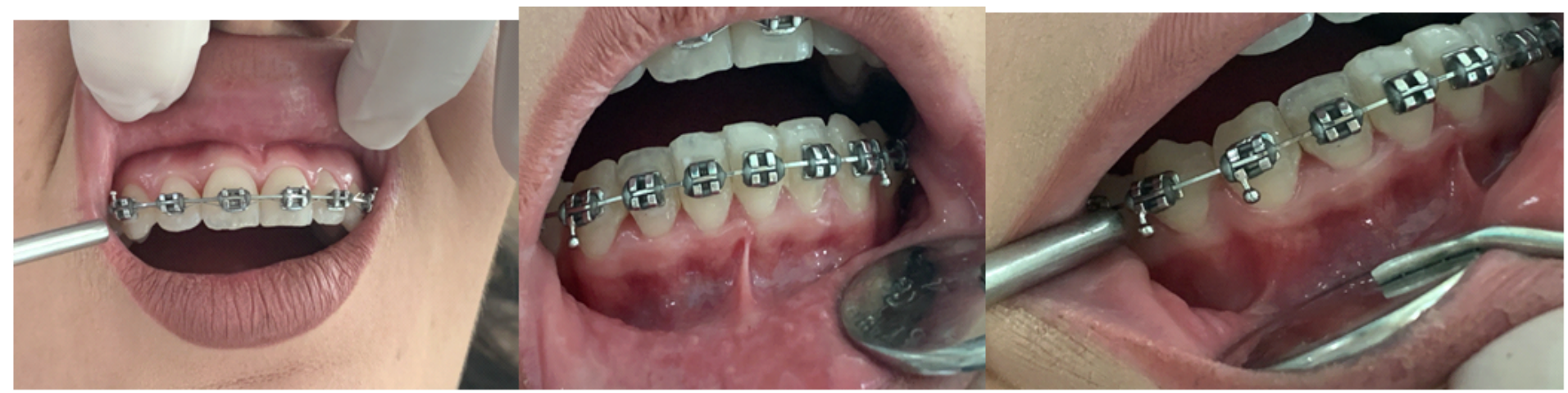

\section{Figure 6}

Photo of patient A. Ç. With fixed orthodontic apparatus where the presence of periodontal stone in the interdental areas of the maxillary and mandibular incisors is distinguished. 


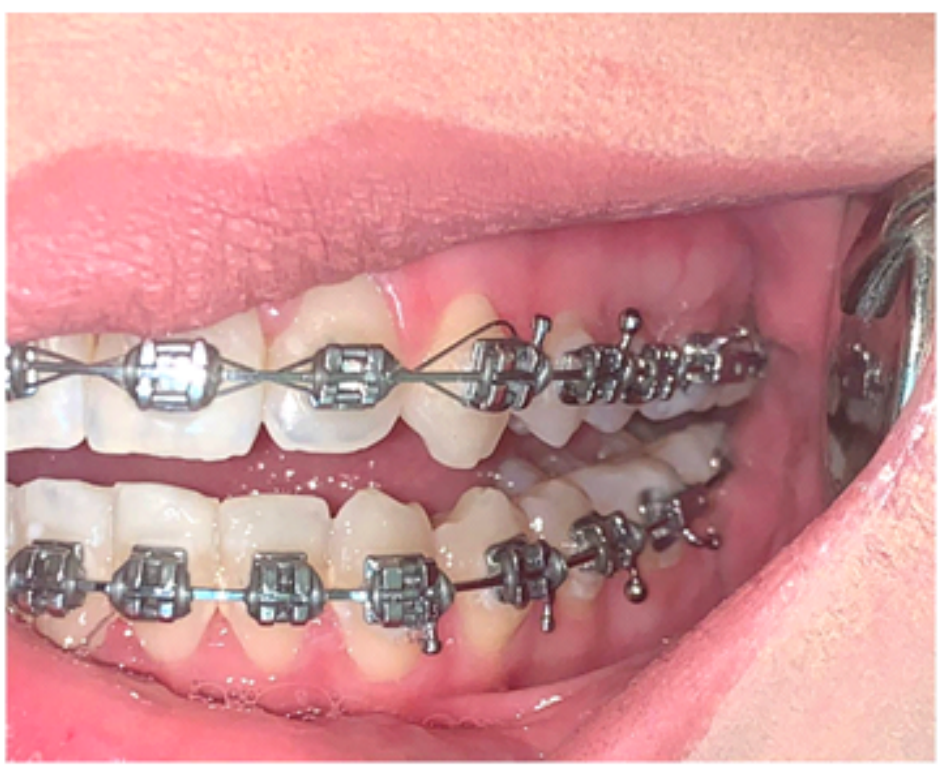

Figure 7

Tendency to gingival hypertrophy in areas 43,44 and 23, 24 .

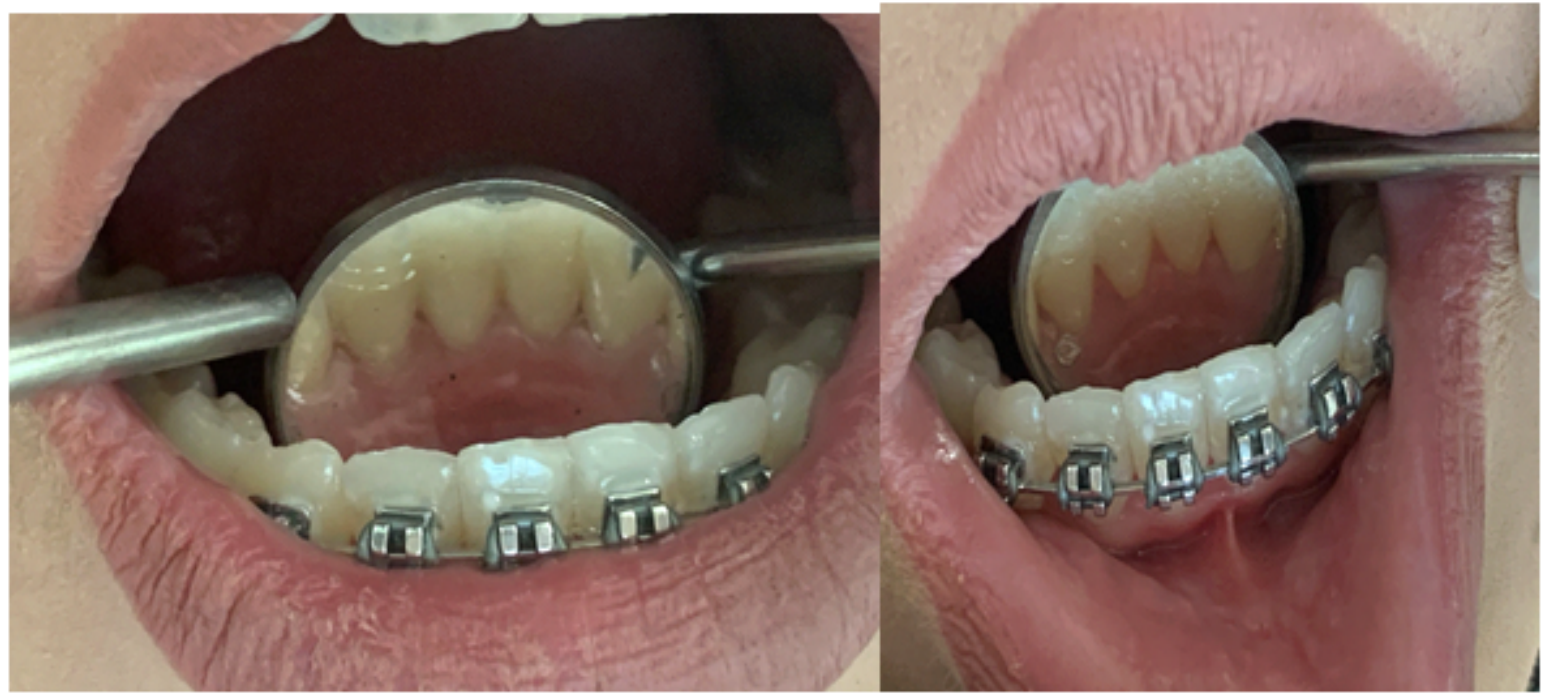

Figure 8

Photo of the lingual surfaces of the manfdibular incisor, showing the presence of stones in the inteproximal surfaces. 

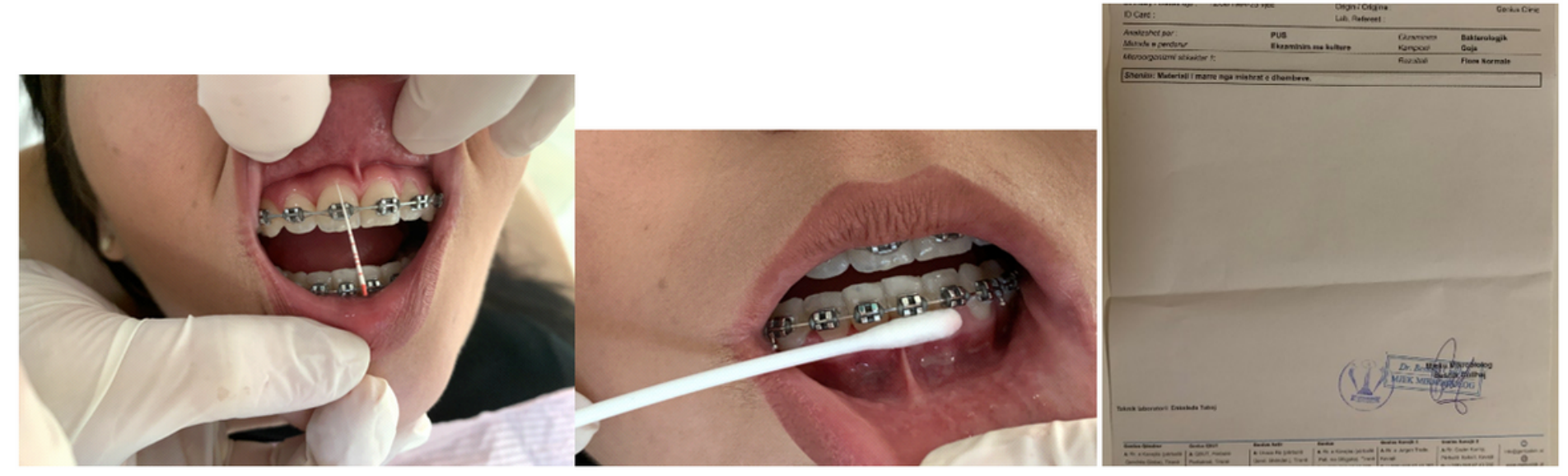

\section{Figure 9}

Photo during the procedure of obtaining material from the gingival sulcus to patient no.2, accompanied by the relevant analysis sheet. 

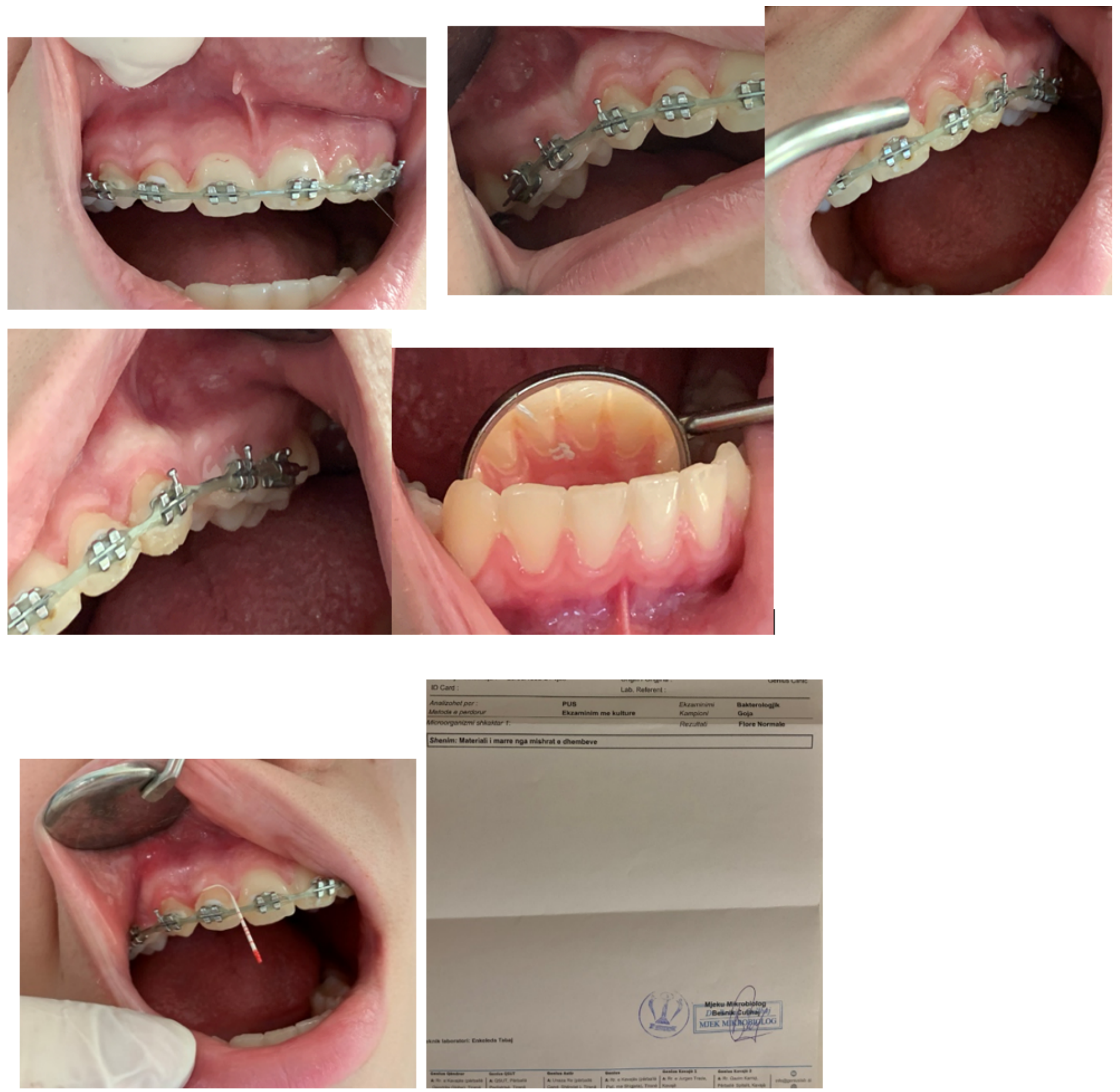

Figure 10

Patient no.3 with data recorded for study effect. 